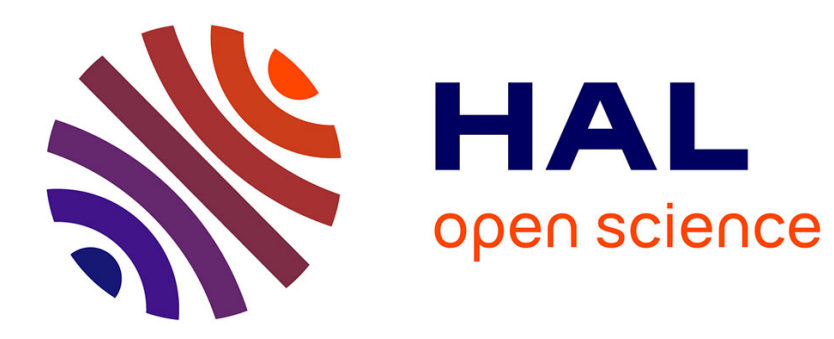

\title{
Ising spin glasses in the effective-interaction-approximation
}

L. Turban, P. Guilmin

\section{To cite this version:}

L. Turban, P. Guilmin. Ising spin glasses in the effective-interaction-approximation. Journal de Physique Lettres, 1980, 41 (7), pp.145-149. 10.1051/jphyslet:01980004107014500 . jpa-00231746

\section{HAL Id: jpa-00231746 https://hal.science/jpa-00231746}

Submitted on 1 Jan 1980

HAL is a multi-disciplinary open access archive for the deposit and dissemination of scientific research documents, whether they are published or not. The documents may come from teaching and research institutions in France or abroad, or from public or private research centers.
L'archive ouverte pluridisciplinaire HAL, est destinée au dépôt et à la diffusion de documents scientifiques de niveau recherche, publiés ou non, émanant des établissements d'enseignement et de recherche français ou étrangers, des laboratoires publics ou privés. 


\title{
LE JOURNAL DE PHYSIQUE-LETTRES
}

J. Physique - LETTRES 41 (1980) L-145- L-149

1 er AVRIL 1980, PAGE L-145

Classification

Physics Abstracts

$75.10 \mathrm{H}$

\section{Ising spin glasses in the effective-interaction-approximation}

\author{
L. Turban and P. Guilmin \\ Laboratoire de Physique du Solide (*), Université de Nancy I Co140, 54037 Nancy Cedex, France
}

(Reçu le 6 novembre 1979, accepté le 7 février 1980)

Résumé. - Les lignes critiques des verres de spin de Harris et d'Ising $( \pm 1)$ sont obtenues dans l'approximation de l'interaction effective. Pour le réseau carré nous retrouvons les résultats exacts de Harris

$$
T_{\mathrm{c}}(\eta) / T_{\mathrm{c}}(0)=1-0,3116 \eta^{2}
$$

et Domany $T_{\mathrm{c}}(p) / T_{\mathrm{c}}(1)=1-3,209(1-p)$. La concentration critique pour le ferromagnétisme $p_{\mathrm{c} 1}$ est en bon accord avec les résultats connus et tend vers sa valeur de champ moyen $p_{\mathrm{c} 1}=1 / 2$ quand $z$, la coordinance du réseau, tend vers l'infini.

Les réseaux non alternés ont une ligne critique rétrograde, typique pour le désordre recuit, dans l'approximation à une liaison. Un développement en amas pour les réseaux triangulaire et nid d'abeilles semble indiquer que ce comportement n'est pas physique dans le cas du désordre trempé.

\begin{abstract}
Using an effective-interaction-approximation we study the critical lines of the Harris and $( \pm 1)$-Ising spin glasses. For the square lattice we recover the exact results of Harris $T_{\mathrm{c}}(\eta) / T_{\mathrm{c}}(0)=1-0.3116 \eta^{2}$ and Domany $T_{\mathrm{c}}(p) / T_{\mathrm{c}}(1)=1-3.209(1-p)$. The critical concentration for ferromagnetism $p_{\mathrm{c} 1}$ is in good agreement with known results and tends to its mean-field value $p_{\mathrm{c} 1}=1 / 2$ when $z$, the coordination number, tends to infinity. Non-alternate lattices present a retrograde critical line, typical of annealed disorder, in the single-bond approximation. A cluster extension of the method on the triangular and honeycomb lattices indicates that this behaviour is unphysical for quenched disorder.
\end{abstract}

One of us (L.T.) recently introduced a new effectivemedium approach to the bond-disorder problem in Ising and Potts systems [1, 2]. This " EffectiveInteraction-Approximation » (EIA) is an extension of the Effective-Medium-Approximation for conduction in mixtures developed by Landauer [3] and Kirkpatrick [4]. In the bond-diluted Ising problem the EIA reproduces the results of Harris [5] for the initial slope of the critical line $T_{\mathrm{c}}(p)$ and gives the correct behaviour $\left(T_{\mathrm{c}}\left(p-p_{\mathrm{c}}\right) \sim\left[\ln \left(p-p_{\mathrm{c}}\right)\right]^{-1}[6]\right)$ near $p_{c}$, the critical concentration for bond percolation; $p_{c}$ itself is exact on the selfdual square lattice, within $1 \%$ of the exact results in two dimensions (2D) and within $5 \%$ of the series results in $3 \mathrm{D}$. The critical line for the bond-diluted Potts model with $s=1$ (percolation limit) on the square lattice is exact.

In this letter we present some results concerning the ferro- (antiferromagnetic) critical line in the Harris model and the $( \pm 1)$-Ising spin glass model where the random interaction strength $K_{i j}=\beta J_{i j}$ is independently distributed on the bonds (ij) according to the two-delta probability distribution :

$$
P\left(K_{i j}\right)=p \delta\left(K_{i j}-K_{\mathrm{a}}\right)+(1-p) \delta\left(K_{i j}+K_{\mathrm{a}}\right) .
$$

The method as it stands is unable to predict any spin glass transition. This should not have much influence on the 2D-results since there is now mounting evidence that for Ising spins the lower critical dimensionality is two [7].

We begin with a presentation of the method; then we give some mean-field results which are valid when the coordination number $z$ tends to infinity; we determine the critical line $T_{\mathrm{c}}(p)$ for the Harris [5] and $( \pm)$-Ising spin glasses and use a cluster extension of the method to show the limitations of the singlebond approximation.

1. Effective-Interaction-Approximation. - Let the nearest-neighbour Ising hamiltonian be written :

$$
-\beta \mathcal{H}=\sum_{(i,)} K_{i j} \sigma_{i} \sigma_{j}+L \sum_{i} \sigma_{i}
$$

where the spin variable $\sigma_{i}$ is equal to \pm 1 and the 
random interaction strength $K_{i j}$ is distributed according to the probability law $P\left(K_{i j}\right)$.

In the EIA, the random system is mapped onto a pure Ising system with $p$ - and nontrivial $T$-dependent

interaction strength $K_{\mathrm{m}}$. The effective-medium (EM) hamiltonian reads :

$$
-\beta \mathcal{H}_{\mathrm{m}}=\sum_{(i j)} K_{\mathrm{m}} \sigma_{i} \sigma_{j}+L \sum_{i} \sigma_{i}
$$

and eq. (2) may be rewritten as :

$$
-\beta \mathcal{H}=-\beta \mathcal{H}_{\mathrm{m}}+\sum_{(i j)} x_{i j} \sigma_{i} \sigma_{j} \quad\left(x_{i j}=K_{i j}-K_{\mathrm{m}}\right) .
$$

The thermal average of any quantity $Q$ is therefore given by :

$$
\langle Q\rangle=\left\langle Q \prod_{(i j)}\left(1+\sigma_{i} \sigma_{j} \tanh x_{i j}\right)\right\rangle_{\mathrm{m}} /\left\langle\prod_{(i j)}\left(1+\sigma_{i} \sigma_{j} \tanh x_{i j}\right)\right\rangle_{\mathrm{m}}
$$

where we used the identity, valid for Ising spins :

$$
\exp \left(x_{i j} \sigma_{i} \sigma_{j}\right) \equiv \cosh x_{i j}\left(1+\sigma_{i} \sigma_{j} \tanh x_{i j}\right)
$$

and where $\langle\ldots\rangle_{\mathrm{m}}$ denotes the EM-thermal average.

In the single-bond approximation :

and :

$$
x_{i j}=x \delta_{i k} \delta_{j l} \quad\left(x=K_{k l}-K_{\mathrm{m}}\right)
$$

$$
\langle Q\rangle=\langle Q\rangle_{\mathrm{m}}+\left[\left\langle Q \sigma_{k} \sigma_{l}\right\rangle_{\mathrm{m}}-\varepsilon_{\mathrm{m}}\langle Q\rangle_{\mathrm{m}}\right] \tanh x /\left(1+\varepsilon_{\mathrm{m}} \tanh x\right) .
$$

In this expression $\varepsilon_{\mathrm{m}}=\left\langle\sigma_{k} \sigma_{l}\right\rangle_{\mathrm{m}}$ is the EM-firstneighbour spin correlation function. After a configurational average we get :

$$
\{\langle Q\rangle\}_{\mathrm{c}}=\langle Q\rangle_{\mathrm{m}}
$$

provided that $Q$ does not involve the random interaction $K_{i j}$ and that the EM-interaction strength $K_{\mathrm{m}}$ is chosen to satisfy :

$$
\int \mathrm{d} K_{k l} P\left(K_{k l}\right) \frac{\tanh \left(K_{k l}-K_{\mathrm{m}}\right)}{1+\varepsilon_{\mathrm{m}} \tanh \left(K_{k l}-K_{\mathrm{m}}\right)}=0 .
$$

As in the single-site CPA, it may be shown that the first nonvanishing corrections to eq. (9) are of the fourth order in $\tanh (x)$ for an interaction-independent $Q$. When $Q$ contains the interaction strength $K_{i j}$, corrections of the second order are to be taken into account.

The effective interaction in the single-bond approximation given by eq. (10) is in fact identical to the exact expression for annealed disorder for which the bonds are allowed to move [8].

As we mentioned above, the method is unable to predict a spin glass transition. Using eq. (8), we can show that the Edwards-Anderson order parameter $q=\left\{\left\langle\sigma_{i}\right\rangle^{2}\right\}_{\mathrm{c}}$ is proportional to $\left\langle\sigma_{i}\right\rangle_{\mathrm{m}}^{2}$, it follows that $q$ is nonzero only when the system is in the ferroor antiferromagnetic state.
2. Mean-field results. - In the mean-field approach the critical interaction strength $K_{\mathrm{c}}$ for a ferromagnetic transition is given by :

$$
z K_{\mathrm{c}}=1
$$

and is exact when $z$ tends to infinity since fluctuations are irrelevant in this limit.

We first consider the effect of bond-dilution on $T_{\mathrm{c}}$. Let $q=1-p$ be the concentration of missing bonds; when $z \rightarrow \infty$ eq. (11) is changed into :

or :

$$
z p K_{\mathrm{c}}(p)=1
$$

$$
T_{\mathrm{c}}(p) / T_{\mathrm{c}}(1)=K_{\mathrm{c}}(1) / K_{\mathrm{c}}(p)=1-q .
$$

In the Harris Ising spin glass [5], $P\left(K_{i j}\right)$ is centred on $K$ :

$$
\begin{aligned}
P\left(K_{i j}\right)=1 / 2\left\{\delta \left[K_{i j}-\right.\right. & K(1+\eta)]+ \\
& \left.+\delta\left[K_{i j}-K(1-\eta)\right]\right\}
\end{aligned}
$$

with $\eta \ll 1$. Then :

$$
z\left[\frac{1}{2} K(1+\eta)+\frac{1}{2} K(1-\eta)\right]=1
$$

and finally :

$$
T_{\mathrm{c}}(\eta) / T_{\mathrm{c}}(0)=1 .
$$

For the ( \pm 1$)$-Ising spin glass (eq. (1)) we get :

$$
z\left[p K_{\mathrm{ac}}(p)-q K_{\mathrm{ac}}(p)\right]=1
$$


so that :

$$
T_{\mathrm{c}}(p) / T_{\mathrm{c}}(1)=1-2 q
$$

In this last case, the ferromagnetic phase disappears $\left(T_{\mathrm{c}}(p)=0\right)$ when $p=p_{\mathrm{c}}=1 / 2$.
3. Harris spin glass in the EIA. - In the Harris spin glass the random interaction $K_{i j}$ is distributed according to eq. (14). Using eq. (10) with $K_{\mathrm{m}}=K_{\mathrm{c}}$, $\varepsilon_{\mathrm{m}}=\varepsilon_{\mathrm{c}}, H=0$, the critical values for the pure Ising system, we get the critical line :

$$
\tanh \left[K_{\mathrm{c}}(\eta)-K_{\mathrm{c}}\right]=\frac{2 \varepsilon_{\mathrm{c}} \tanh ^{2} \eta K_{\mathrm{c}}(\eta)}{1-\tanh ^{2} \eta K_{\mathrm{c}}(\eta)+\left[\left(\tanh ^{2} \eta K_{\mathrm{c}}(\eta)-1\right)^{2}+4 \varepsilon_{\mathrm{c}}^{2} \tanh ^{2} \eta K_{\mathrm{c}}(\eta)\right]^{1 / 2}}
$$

In the limit of small fluctuations $(\eta \ll 1)$, expending $K_{\mathrm{c}}(\eta)$ near $K_{\mathrm{c}}$ leads to :

$$
T_{\mathrm{c}}(\eta) / T_{\mathrm{c}}(0)=1-a_{\mathrm{c}} \eta^{2}
$$

with :

$$
a_{\mathrm{c}}=K_{\mathrm{c}} \varepsilon_{\mathrm{c}} \text {. }
$$

This coefficient $a_{\mathrm{c}}$ for 2D and 3D lattices is given in table I, where it can be seen that the results of Harris [5] are recovered for the square and cubic lattices. We observe that when $z$ increases, $a_{\mathrm{c}}$ tends to the meanfield value $a_{\mathrm{c}}=0$ (eq. (15)).

\section{4. ( \pm 1$)$-Ising spin glass in the single-bond EIA. -} For the $( \pm 1)$-Ising spin glass, the critical line is obtained as above using eqs. (1) and (10) and the equation determining $K_{\mathrm{ac}}(p)$ reads :

$\tanh K_{\mathrm{ac}}(p)=$

with :

$$
= \pm \frac{(2 p-1)\left(1-\tanh ^{2} K_{\mathrm{c}}\right) \pm \Delta\left(p, K_{\mathrm{c}}\right)^{1 / 2}}{2\left(\left|\varepsilon_{\mathrm{c}}\right|-\tanh \left|K_{\mathrm{c}}\right|\right)}
$$

$$
\begin{array}{r}
\Delta\left(p, K_{\mathrm{c}}\right)=\left(1+\tanh ^{2} K_{\mathrm{c}}-2 \varepsilon_{\mathrm{c}} \tanh K_{\mathrm{c}}\right)^{2}- \\
-4 p(1-p)\left(1-\tanh ^{2} K_{\mathrm{c}}\right)^{2} .
\end{array}
$$

The critical concentration

$$
p_{\mathrm{c} 1}=\left(1+\varepsilon_{\mathrm{c}}\right) / 2
$$

is deduced from eq. (22) with $\tanh \left(K_{\mathrm{ac}}(p)\right)=1$.

The critical concentration for ferromagnetism $p_{\mathrm{c} 1}$ is given in table I for the different lattices. The square lattice result is in good agreement with recent percolation estimates (Domany [9] $p_{\mathrm{c} 1}=0.826$; Vannimenus and Toulouse [10] $p_{\mathrm{c} 1}=0.91$ ) and Monte Carlo results (Kirkpatrick [11] $p_{\mathrm{c} 1}=0.80-0.85$; Ono [12] $\left.p_{\mathrm{c} 1}=0.85\right)$. The behaviour of $p_{\mathrm{c} 1}$ with increasing $z$ is also correctly predicted.

The properties of the critical line near $p=1$ can be determined using eq. (22). The expansion of $K_{\mathrm{ac}}(p)$ near $K_{\mathrm{c}}$ leads to :

with :

$$
T_{\mathrm{c}}(p) / T_{\mathrm{c}}(1)=1-b_{\mathrm{c}}(1-p)
$$

$$
b_{\mathrm{c}}^{-1}=K_{\mathrm{c}}\left(\operatorname{coth} K_{\mathrm{c}}+\tanh K_{\mathrm{c}}-2 \varepsilon_{\mathrm{c}}\right)
$$

where $b_{c}$ is given in table I for 2D and 3D lattices. For the square lattice, we recover an "exact result of Domany [9], obtained through a variational calculation. When $z$ increases, $b_{c}$ tends to its mean-field value, $b_{\mathrm{c}}=2$ (eq. (17)), as expected.

Some of the features of the single-bond approximation are typical for annealed systems where the

Table I. - Normalized initial slope of the critical line near $p=1$ for 2D and 3D lattices in the Harris spin glass $\left(a_{\mathrm{c}}\right)$ and $(\mp 1)$-Ising spin glass $\left(b_{\mathrm{c}}\right)$. Critical concentrations for ferromagnetism in the single-bond approximation $\left(p_{\mathrm{c} 1}\right)$ and three-bonds approximation $\left(p_{\mathrm{c} 3}\right)$ for the $( \pm 1)$-Ising spin glass.

$\begin{array}{cccccccc}\text { Lattices } & = & K_{\mathrm{c}}[16] & \varepsilon_{\mathrm{c}}[16] & a_{\mathrm{c}} & b_{\mathrm{c}} & p_{\mathrm{c} 1} & p_{\mathrm{c} 3} \\ - & - & - & - & - & - & - & 0.908 \\ \text { Honeycomb } & 3 & 0.6585 & 0.7698 & 0.5069 & 3.946 & 0.885 & - \\ \text { Kagomé } & 4 & 0.4666 & 0.7440 & 0.3472 & 3.445 & 0.8720 & - \\ \text { Square } & 4 & 0.4407 & 0.7071 & 0.3116 & 3.209 & 0.854 & - \\ \text { Dice } & 4 & 0.4157 & 0.6683 & 0.2778 & 3.008 & 0.834 & - \\ \text { Triangular } & 6 & 0.2747 & 0.6667 & 0.1831 & 2.731 & 0.833 & 0.806 \\ \text { Diamond } & 4 & 0.370 & 0.432 & 0.160 & 2.334 & 0.716 & - \\ \text { SC } & 6 & 0.2217 & 0.3284 & 0.0728 & 2.177 & 0.664 & - \\ \text { BCC } & 8 & 0.158 & 0.270 & 0.043 & 2.103 & 0.635 & - \\ \text { FCC } & 12 & 0.102 & 0.245 & 0.025 & 2.076 & 0.623 & 0.624 \\ \text { Mean-field } & x & - & - & 0 & 2 & 1 / 2 & -\end{array}$


bonds are allowed to move in order to minimize the free energy. The line of maximum magnetization in the ferromagnetic phase given by :

$$
\Delta\left(p, K_{\mathrm{m}}\right)=0
$$

and the occurrence of multiple transitions (retrograde critical line) on non-alternate lattices (Fig. 1b) are not recovered in the three-bonds approximation given below. The retrograde critical line in annealed systems has been discussed by Kasai and Syosi [13].

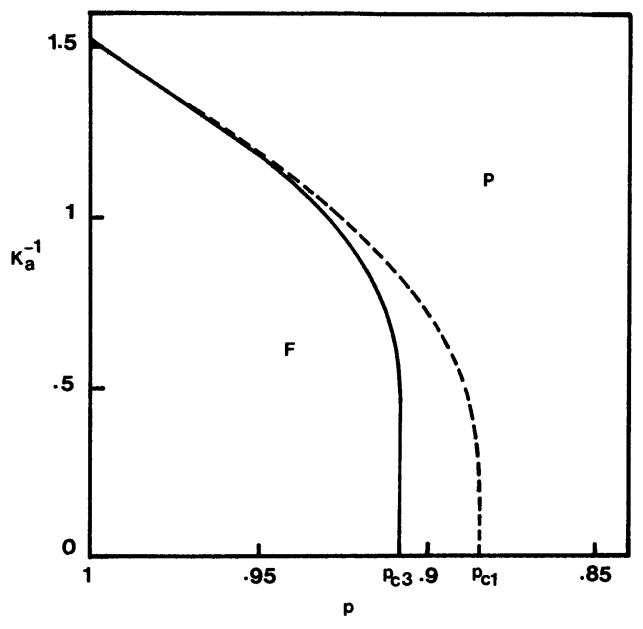

[a]

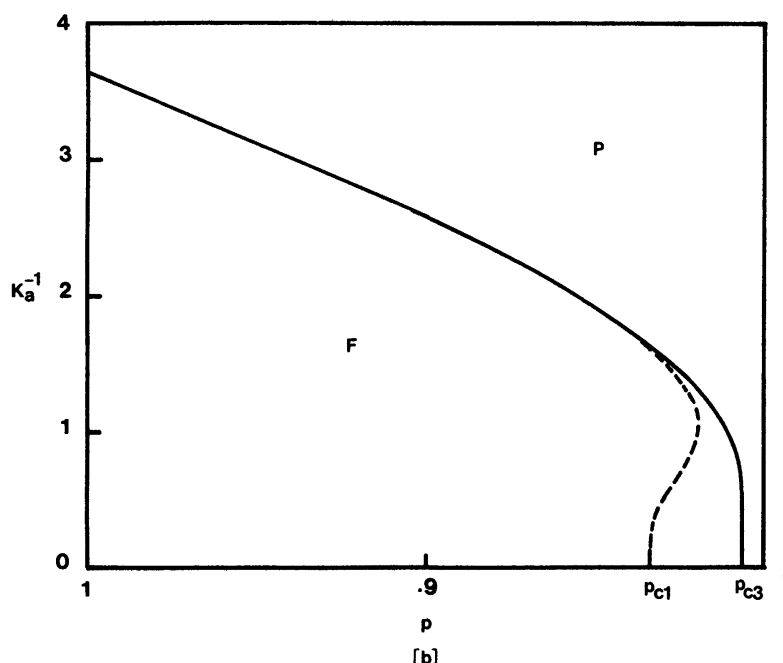

Fig. 1. - Critical line for ferromagnetism in the Ising spin glass (single-bond approximation : dashed line; three-bonds approximation : heavy line) for the honeycomb lattice $(a)$ and the triangular lattice $(b)$.

5. ( \pm 1 )-Ising spin glass in the three-bonds approximation. - The EIA is easily extended beyond the single-bond approximation by working on a cluster. We illustrate this possibility using a three-bonds cluster on the triangular and honeycomb lattices. The treatment is parallel to that used by one of us in the conduction problem [14].

Eq. (5) remains valid but the perturbation on the effective medium $x_{i j}$ is now nonzero on the three- bonds of the cluster which is chosen in agreement with the symmetry of the lattice (Fig. 2a,b). A simple expression like eq. (8) for any $Q$ is no longer obtained and one has to specify $Q$. This is done here using :

$$
Q=\sigma_{i} \sigma_{j}
$$

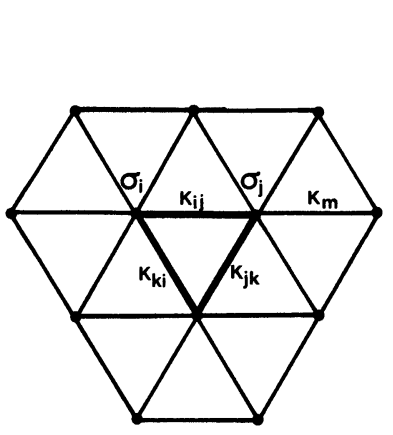

[a]

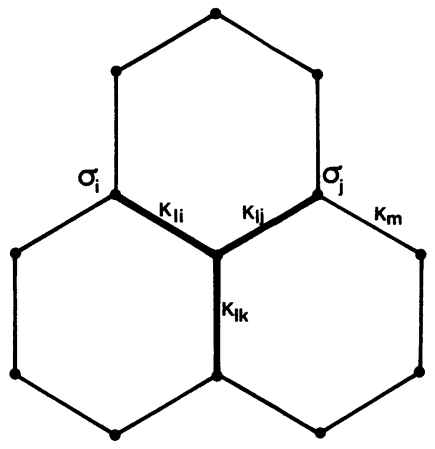

[b]
Fig. 2. - Three-bonds clusters used in the three-bonds approximation on the triangular $(a)$ and honeycomb $(b)$ lattices.

where $\sigma_{i}$ and $\sigma_{j}$ are any two- of the three-spins common to the cluster and the effective medium. $\sigma_{i} \sigma_{j}$ plays the same role in this problem as the potential difference $\left(V_{i}-V_{j}\right)$ in the conduction problem [14]. The effective interaction is obtained by requiring that :

$$
\left\{\left\langle\sigma_{i} \sigma_{j}\right\rangle\right\}_{\mathrm{c}}=\left\langle\sigma_{i} \sigma_{j}\right\rangle_{\mathrm{m}} .
$$

This equation involves only the first-neighbour spin correlation function $\varepsilon_{1}$, on the triangular lattice, whereas second-neighbours and four-spins correlation functions $\varepsilon_{2}$ and $\varepsilon_{4}$ are needed on the honeycomb lattice. These are easily obtained through duality and a star-triangle transformation (details will be given elsewhere) and reads :

$$
\varepsilon_{2 \mathrm{c}}=2 / 3 \quad \varepsilon_{4 \mathrm{c}}=1 / \sqrt{3} .
$$

The critical lines (Fig. 1 $a, b$ ) are obtained as above and we get the critical concentrations :

$p_{\mathrm{c} 3}($ honeycomb $)=0.908$;

$$
p_{\mathrm{c} 3}(\text { triangular })=0.806
$$

These values are within $2-3 \%$ of the single-bond results. On the honeycomb lattice $p_{\mathrm{c} 3}>p_{\mathrm{c} 1}$ whereas the reverse is true on the triangular lattice. On intuitive ground, one would expect $p_{\mathrm{c}}$, critical concentration for quenched disorder, to be greater than $p^{*}$, critical concentration for annealed disorder, or $p_{\mathrm{c} 3}>p_{\mathrm{c} 1}$ since $p_{\mathrm{c} 1}=p^{*}$. In fact this inequality is not always satisfied in the problem of bond dilution where exact results are known $[15,16]$ (Honeycomb : $p_{\mathrm{c}}=0.6527$, $p^{*}=0.6478$; triangular : $p_{\mathrm{c}}=0.3473, p^{*}=0.3522$ ). Notice that for both problems we have $p_{\mathrm{c}}<p^{*}$ on the triangular lattice. 


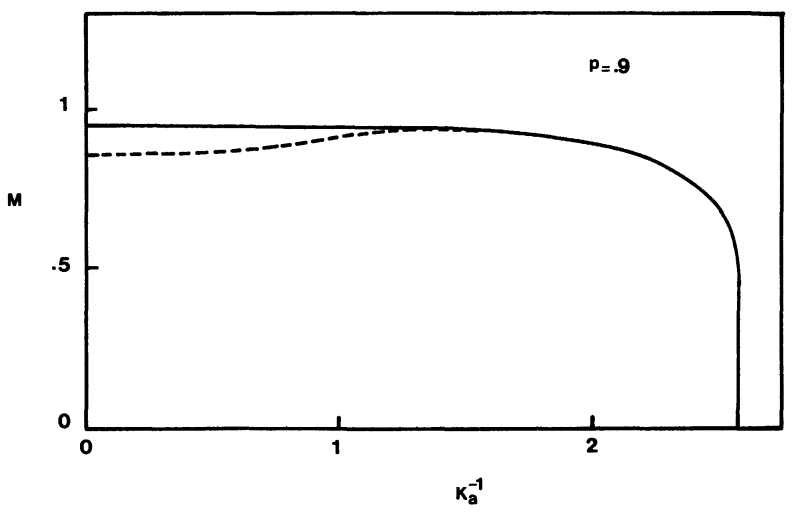

[a]

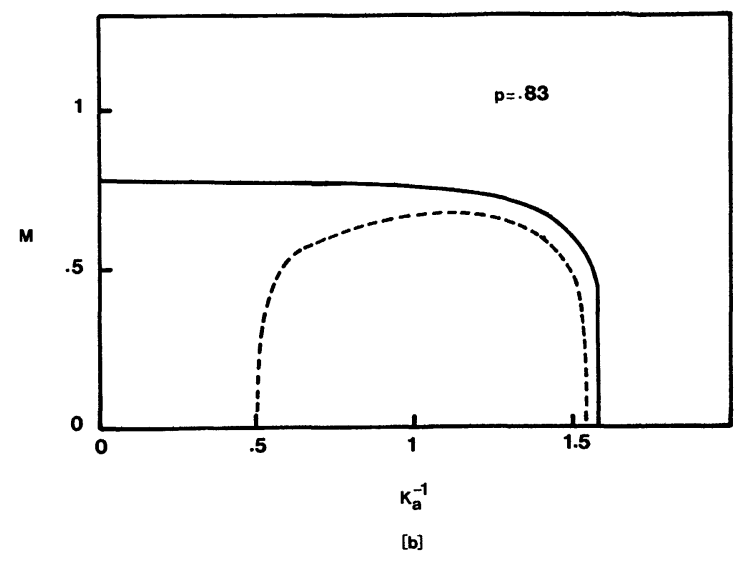

Fig. 3. - Spontaneous magnetization of the Ising spin glass in the single-bond approximation (dashed line) and three-bonds approximation (heavy line) for positive-bonds concentrations $p=0.9(a)$ and $p=0.83(b)$.

In the three-bonds approximation the critical line is no longer retrograde on the triangular lattice. The spontaneous magnetization was also calculated (Fig. $3 a, b$ ) and the line of maximum magnetization at finite temperature, which is observed in annealed systems [13], no longer occur in the three-bonds approximation for both lattices.

The difference between quenched and annealed disorder is only taken into account when working on a cluster with more than one bond so that we expect these qualitative changes between the two approximations to be significant.

To end let us mention that in the bond dilution problem exact results for the bond percolation thresholds are obtained on the triangular and honeycomb lattices giving us some confidence in the reliability of the three-bonds approach in the low-temperature range.

\section{References}

[1] Turban, L., Phys. Lett. A (1980) (to be published). Similar approaches were proposed earlier by HARRIS, A. B., J. Phys. C 9 (1976) 2515 and Lage, E. J. S., J. Phys. C 10 (1977) 701.

[2] Turban, L., J. Phys. C 13 (1980) (to be published).

[3] Landauer, R., J. Appl. Phys. 23 (1952) 779.

[4] Kirk Patrick, S., Rev. Mod. Phys. 45 (1973) 574.

[5] Harris, A. B., J. Phys. C 7 (1974) 1671.

[6] Bergstresser, T. K., J. Phys. C 10 (1977) 3831.

[7] Anderson, P. W. and Pond, C. M., Phys. Rev. Lett. 40 (1978) 903.

[8] Thorpe, M. F. and Beeman, D., Phys. Rev. B 14 (1976) 188, eq. (13).
[9] Domany, E., J. Phys. C 12 (1979) L119.

[10] Vannimenus, J. and Toulouse, G., J. Phys. C 10 (1977) L537.

[11] KiRkPatrick, S., Phys. Rev. B 16 (1977) 4630

[12] Ono, I., J. Phys. Soc. Japan 41 (1976) 345.

[13] Kasai, Y. and Syosi, I., Prog. Theor. Phys. 50 (1973) 1182.

[14] Turban, L., J. Phys. C 11 (1978) 449.

[15] Sykes, M. F. and Essam, J. W., J. Math. Phys. 5 (1964) 1117.

[16] Syosi, I., Phase transitions and critical phenomena, Vol. I, C. Domb and M. S. Green eds. (London : Academic Press) 1972, p. 269. 